Commun. Korean Math. Soc. 27 (2012), No. 1, pp. 175-183

http://dx.doi.org/10.4134/CKMS.2012.27.1.175

\title{
ON SOME COMBINATIONS OF SELF-RECIPROCAL POLYNOMIALS
}

\author{
SEON-Hong KIM
}

\begin{abstract}
Let $\mathcal{P}_{n}$ be the set of all monic integral self-reciprocal polynomials of degree $n$ whose all zeros lie on the unit circle. In this paper we study the following question: For $P(z), Q(z) \in \mathcal{P}_{n}$, does there exist a continuous mapping $r \rightarrow G_{r}(z) \in \mathcal{P}_{n}$ on $[0,1]$ such that $G_{0}(z)=P(z)$ and $G_{1}(z)=Q(z)$ ?
\end{abstract}

\section{Introduction}

Throughout this paper, $U$ denotes the unit circle and $n$ is a positive integer. If all zeros of $P(z) \in \mathbb{R}[z]$ of degree $n$ lie on $U$, it is well known that $P(z)=$ $\pm z^{n} P(1 / z)$, and $P(z)$ is called self-reciprocal when $P(z)=z^{n} P(1 / z)$. In what follows we denote by $\mathcal{P}_{n}$ the set of all monic integral self-reciprocal polynomials of degree $n$ whose all zeros lie on $U$. Our basic goal in this paper is the study of the following question.

Question A. For given polynomials $P(z), Q(z) \in \mathcal{P}_{n}$, does there exist a continuous mapping $r \rightarrow G_{r}(z) \in \mathcal{P}_{n}$ on $[0,1]$ such that

$$
G_{0}(z)=P(z), \quad G_{1}(z)=Q(z) ?
$$

The condition $P(z)$ and $Q(z)$ self-reciprocal in Question A seems to be necessary because $z+1$ and $z-1$ are the only monic integral polynomials of degree 1 with all zeros on $U$ and so no $G_{r}(z)$ in Question A exists. One may ask naturally whether $G_{r}(z)$ in Question A is the convex combination of $P(z)$ and $Q(z)$, that is $G_{r}(z)=(1-r) P(z)+r Q(z)$ where $0 \leq r \leq 1$. However $G_{r}(z)$ is not always the convex combination, for example the polynomials

$$
\begin{aligned}
& P(z)=\left(z^{2}-z+1\right)\left(z^{4}+1\right)\left(z^{6}+z^{5}+z^{4}+z^{3}+z^{2}+z+1\right), \\
& Q(z)=\left(z^{4}-z^{3}+z^{2}-z+1\right)\left(z^{8}-z^{6}+z^{4}-z^{2}+1\right)
\end{aligned}
$$

Received October 15, 2010.

2010 Mathematics Subject Classification. Primary 30C15; Secondary 26C10.

Key words and phrases. convex combination, polynomials, self-reciprocal polynomials, unit circle, zeros.

This research was supported by Basic Science Research Program through the National Research Foundation of Korea(NRF) funded by the Ministry of Education, Science and Technology (No.2009-0075759). 
have all their zeros on $U$, but $(P(z)+Q(z)) / 2$ has four zeros of modulus $1.15963 \cdots$. This yields a question about the existence of $G_{r}(z)$ which is not the convex combination. If Question $\mathrm{A}$ is true, we will write

$$
P \sim Q,
$$

and specially if $G_{r}(z)$ is the convex combination, we will write

$$
P \hookrightarrow Q
$$

Preliminary attempts using computer algebra lead to the various unproved examples including

$$
\frac{z^{2 n+1}-1}{z-1} \hookrightarrow\left(\frac{z^{n+1}-1}{z-1}\right)^{2}
$$

and

$$
\left(\frac{z^{n}-1}{z-1}\right)^{3} \hookrightarrow z^{3(n-1)}+z^{3(n-1)-1}+\cdots+1
$$

And Kim [2] showed

$$
\left(z^{2 n}+1\right)^{2} \sim\left(z^{2}-1\right)\left(z^{4 n-2}-1\right)
$$

for some $G_{r}(z)$ that is not the convex combination. But it seems to be hard to find such $G_{r}(z)$.

In Section 2, we will give examples of self-reciprocal polynomials $P(z)$ and $Q(z)$ satisfying $P \sim Q$ in different two ways and one of them is $P \hookrightarrow Q$. This generalizes Theorem 1 of [2]. In Section 3, we will consider self-reciprocal polynomials that are products of finite geometric series. Let

$$
F_{a_{1}, \ldots, a_{u}}(z)=\prod_{j=1}^{u} \frac{z^{a_{j}}-1}{z-1}
$$

$\mathcal{F}_{u, n}=\left\{F_{a_{1}, \ldots, a_{u}}(z):\right.$ all $a_{j} \in \mathbb{N}-\{1\}(1 \leq j \leq u)$ are distinct, $\left.\sum_{j=1}^{u} a_{j}=n\right\}$.

We will prove a conjecture given in [2] that $f_{1} \hookrightarrow f_{2}$ for any $f_{1}, f_{2} \in \mathcal{F}_{2, n}$. In case of $u \geq 3$, not all $g_{1}, g_{2} \in \mathcal{F}_{u, n}$ satisfy $g_{1} \hookrightarrow g_{2}$. But we will see that, using the case $u=2$, for any $g_{1}, g_{3} \in \mathcal{F}_{3, n}$, there exists $g_{2} \in \mathcal{F}_{3, n}$ such that $g_{1} \hookrightarrow g_{2} \hookrightarrow g_{3}$, and such $g_{2}$ is not unique. Also we will study how to show $g_{1} \hookrightarrow g_{2}$ where $g_{1}, g_{2} \in \mathcal{F}_{3, n}$ are specifically given. The main tool for this is Fell's lemma [1]. Finally in Section 4, we will discuss whether " $\sim$ " is an equivalence relation or not over the set $\mathcal{F}_{u, n}$. It is obvious that " $\sim$ " is reflexive and symmetric. But we don't give an answer for transitivity but at least we will see that a natural candidate for this does not work. 


\section{2. $P \sim Q$ in two ways}

In this section, we show that some $P(z)$ and $Q(z)$ satisfy both $P \hookrightarrow Q$ and $P \sim Q$ as not a convex combination. This generalizes Theorem 1 of [2]. Using Cohn's theorem (see p. 230 of [3]) we prove:

Theorem 1. Let $a, b, c \in \mathbb{N}$ with $c>a>b$ and $2 a=b+c$. Then we have

(a) $\left(z^{a} \pm 1\right)^{2} \hookrightarrow\left(z^{b}-1\right)\left(z^{c}-1\right)$,

(b) $\left(z^{a} \pm 1\right)^{2} \sim\left(z^{b}-1\right)\left(z^{c}-1\right)$,

where

$$
G_{r}(z)=z^{2 a}-r^{a} z^{c}-\left( \pm r^{c} \pm r^{b} \mp 2\right) z^{a}-r^{a} z^{b}+1, \quad 0 \leq r \leq 1 .
$$

Proof. Suppose that $a, b, c \in \mathbb{N}$ with $c>a>b, 2 a=b+c$, and $0<r<1$. We first prove (a). Write $G_{0}(z)=\left(z^{a} \pm 1\right)^{2}, G_{1}(z)=\left(z^{b}-1\right)\left(z^{c}-1\right)$ and

$$
\begin{aligned}
G_{r}(z) & =(1-r) G_{0}(z)+r G_{1}(z) \\
& =z^{2 a}-r z^{c}+2( \pm 1 \mp r) z^{a}-r z^{b}+1 .
\end{aligned}
$$

Then we can compute

$$
H_{r}(z):=\frac{G_{r}^{\prime}(z)}{z^{b-1}}=2 a z^{2 a-b}-c r z^{c-b}+2 a( \pm 1 \mp r) z^{a-b}-b r .
$$

Define, for $\epsilon>0$,

$$
H_{r, \epsilon}(z):=(2 a+\epsilon) z^{2 a-b}-c r z^{c-b}+2 a( \pm 1 \mp r) z^{a-b}-b r .
$$

For $|z|=1$,

$$
\left|H_{r, \epsilon}(z)\right| \geq 2 a+\epsilon-(c r+2 a(1-r)+b r)=\epsilon>0
$$

which implies $H_{r, \epsilon}(z)$ does not have a zero on $U$. By Roché, $H_{r, \epsilon}(z)$ has all $2 a-b$ zeros strictly inside $U$. Letting $\epsilon \rightarrow 0$, we see that $G_{r}^{\prime}(z)$ has all its zeros inside or on $U$. It follows from Cohn's theorem that the proof of (a) is complete. The proof of (b) is very similar to the above. We again let $G_{0}(z)=\left(z^{a} \pm 1\right)^{2}$ and $G_{1}(z)=\left(z^{b}-1\right)\left(z^{c}-1\right)$. From (1) we may calculate

$$
\frac{G_{r}^{\prime}(z)}{z^{b-1}}=2 a z^{2 a-b}-c r^{a} z^{c-b}-a\left( \pm r^{c} \pm r^{b} \mp 2\right) z^{a-b}-b r^{a} .
$$

Let

$$
\begin{aligned}
& f(z)=2 a z^{2 a-b} \\
& g(z)=-c r^{a} z^{c-b}-a\left( \pm r^{c} \pm r^{b} \mp 2\right) z^{a-b}-b r^{a} .
\end{aligned}
$$

On $|z|=1$,

$$
\begin{aligned}
|g(z)| & \leq c r^{a}+a\left(2-r^{c}-r^{b}\right)+b r^{a} \\
& =2 a r^{a}+2 a-a\left(r^{c}+r^{b}\right) \\
& =a\left(2 r^{a}+2-r^{c}-r^{b}\right) \\
& <2 a=|f(z)| .
\end{aligned}
$$


Last inequality follows from the arithmetic-geometric mean inequality, that is

$$
\frac{r^{c}+r^{b}}{2}>\sqrt{r^{c+b}}=r^{a} .
$$

Hence, by Rouché, $G_{r}^{\prime}(z)$ has all its zeros inside $U$. It follows from Cohn's theorem that the proof is complete.

\section{Products of finite geometric series}

In Section 3, we consider self-reciprocal polynomials that are products of finite geometric series. With recall

$$
F_{a_{1}, \ldots, a_{u}}(z)=\prod_{j=1}^{u} \frac{z^{a_{j}}-1}{z-1}
$$

$\mathcal{F}_{u, n}=\left\{F_{a_{1}, \ldots, a_{u}}(z):\right.$ all $a_{j} \in \mathbb{N}-\{1\}(1 \leq j \leq u)$ are distinct, $\left.\sum_{j=1}^{u} a_{j}=n\right\}$,

we prove a conjecture given in [2] which aserts $f_{1} \hookrightarrow f_{2}$ for any $f_{1}, f_{2} \in \mathcal{F}_{2, n}$. The proof of this is similar to that of Theorem 1. Multiplying $(z-1)^{2}$ of each side, we only need to show:

Theorem 2. Let $a, b, c, d \in \mathbb{N}$ with not all equal and $a+b=c+d$. Then we have

$$
\left(z^{a}-1\right)\left(z^{b}-1\right) \hookrightarrow\left(z^{c}-1\right)\left(z^{d}-1\right) .
$$

Proof. We may assume that $c>a>b>d$. Write

$$
\begin{aligned}
& G_{0}(z)=\left(z^{a}-1\right)\left(z^{b}-1\right)=z^{a+b}-z^{a}-z^{b}+1, \\
& G_{1}(z)=\left(z^{c}-1\right)\left(z^{d}-1\right)=z^{c+d}-z^{c}-z^{d}+1,
\end{aligned}
$$

and for $0<r<1$,

$$
\begin{aligned}
G_{r}(z) & =(1-r) G_{0}(z)+r G_{1}(z) \\
& =z^{c+d}-r z^{c}-(1-r) z^{a}-(1-r) z^{b}-r z^{d}+1 .
\end{aligned}
$$

Then we can compute

$$
H_{r}(z):=\frac{G_{r}^{\prime}(z)}{z^{d-1}}=(c+d) z^{c}-c r z^{c-d}-a(1-r) z^{a-d}-b(1-r) z^{b-d}-d r .
$$

Define, for $\epsilon>0$,

$$
H_{r, \epsilon}(z):=(c+d+\epsilon) z^{c}-c r z^{c-d}-a(1-r) z^{a-d}-b(1-r) z^{b-d}-d r .
$$

For $|z|=1$,

$$
\left|H_{r, \epsilon}(z)\right| \geq c+d+\epsilon-(c r+a(1-r)+b(1-r)+d r)=\epsilon>0
$$

which implies $H_{r, \epsilon}(z)$ does not have a zero on $U$. By Roché, $H_{r, \epsilon}(z)$ has all $c$ zeros strictly inside $U$. Letting $\epsilon \rightarrow 0$, we see that $G_{r}^{\prime}(z)$ has all its zeros inside or on $U$. It follows from Cohn's theorem that the proof is complete. 
Unlikely for $u=2$, not all $g_{1}, g_{2} \in \mathcal{F}_{u, n}, u \geq 3$ satisfy $g_{1} \hookrightarrow g_{2}$. For example,

$$
F_{3,7,10} \hookrightarrow F_{4,5,11}
$$

because $F_{3,7,10}+F_{4,5,11}$ has four zeros not on $U$. But using the case $u=2$, we show:

Proposition 3. For any $g_{1}, g_{3} \in \mathcal{F}_{3, n}$, there exists $g_{2} \in \mathcal{F}_{3, n}$ such that $g_{1} \hookrightarrow$ $g_{2} \hookrightarrow g_{3}$.

Proof. Let $F_{a_{1}, a_{2}, a_{3}}, F_{c_{1}, c_{2}, c_{3}} \in \mathcal{F}_{3, n}$ with

$$
\min \left\{a_{1}, a_{2}, a_{3}\right\}=a_{1} \text { and } \min \left\{c_{1}, c_{2}, c_{3}\right\}=c_{1} .
$$

If $a_{i}=c_{j}$ for some $i$ and $j$, we take $b_{1}=a_{i}=c_{j}$ so that, by Theorem 2, $F_{a_{1}, a_{2}, a_{3}} \hookrightarrow F_{b_{1}, b_{2}, b_{3}}$ and $F_{c_{1}, c_{2}, c_{3}} \hookrightarrow F_{b_{1}, b_{2}, b_{3}}$ for some $b_{2}$ and $b_{3}$. Assume that none of $a_{i}, c_{j}$ are same. Since $2 \leq a_{1}, c_{1} \leq\left[\frac{n}{3}\right]-1$, we have

$$
2 \leq a_{1}+c_{1} \leq \frac{2 n}{3}-2<n
$$

Choose $b_{1}=a_{1}$ and $b_{2}=c_{1}$ so that $b_{1}+b_{2}<n$. By Theorem 2 again, $F_{a_{1}, a_{2}, a_{3}} \hookrightarrow F_{b_{1}, b_{2}, b_{3}}$ and $F_{c_{1}, c_{2}, c_{3}} \hookrightarrow F_{b_{1}, b_{2}, b_{3}}$ for some $b_{2}$ and $b_{3}$.

The $g_{2}$ of above theorem is not unique. For example, we may check that

$$
\begin{aligned}
& F_{3,7,10} \hookrightarrow F_{5,6,9} \hookrightarrow F_{4,5,11}, \\
& F_{3,7,10} \hookrightarrow F_{2,7,11} \hookrightarrow F_{4,5,11}, \\
& F_{3,7,10} \hookrightarrow F_{2,8,10} \hookrightarrow F_{4,5,11} .
\end{aligned}
$$

Next we will provide an example about the way to show $g_{1} \hookrightarrow g_{2}$ where $g_{1}, g_{2} \in$ $\mathcal{F}_{3, n}$ are specifically given. The main tool for this is Fell's lemma [1] below.

Lemma 4 (Fell). Let $P_{0}(z)$ and $P_{1}(z)$ be real monic polynomials of degree $n$ with their zeros contained in the unit circle except for -1 and 1 . Denote the zeros of $P_{0}(z)$ by $w_{1}, w_{2}, \ldots, w_{n}$ and of $P_{1}(z)$ by $z_{1}, z_{2}, \ldots, z_{n}$. Assume that

$$
w_{i} \neq z_{j} \quad(1 \leq i, j \leq n)
$$

and

$$
\begin{aligned}
& 0<\arg \left(w_{i}\right) \leq \arg \left(w_{j}\right)<2 \pi, \\
& 0<\arg \left(z_{i}\right) \leq \arg \left(z_{j}\right)<2 \pi \quad(1 \leq i<j \leq n) .
\end{aligned}
$$

Let $\alpha_{i}$ be the smaller open arc of the unit circle bounded by $w_{i}$ and $z_{i}(i=$ $1, \ldots, n)$. Then the locus of zeros of $(1-A) P_{0}(z)+A P_{1}(z)(0 \leq A \leq 1)$ is contained in the unit circle if and only if the arcs $\alpha_{i}$ are disjoint.

For $F_{a_{1}, a_{2}, a_{3}}, F_{b_{1}, b_{2}, b_{3}} \in \mathcal{F}_{3, n}$, write

$$
c_{j}=\max _{1 \leq i \leq 3} \operatorname{gcd}\left(a_{i}, b_{j}\right), \quad 1 \leq j \leq 3,
$$


and if $c_{j_{k}}=c_{j_{l}}$ we convention that one of these equals 1 . Then $F_{a_{1}, a_{2}, a_{3}}$ and $F_{b_{1}, b_{2}, b_{3}}$ have common factor

$$
\prod_{j=1}^{3}\left(z^{c_{j}-1}+z^{c_{j}-2}+\cdots+1\right)
$$

and hence to show $F_{a_{1}, a_{2}, a_{3}} \hookrightarrow F_{b_{1}, b_{2}, b_{3}}$, we apply above lemma to the integral polynomial

$$
P_{r}(z):=\frac{(1-r) F_{a_{1}, a_{2}, a_{3}}+r F_{b_{1}, b_{2}, b_{3}}}{\prod_{j=1}^{3}\left(z^{c_{j}-1}+z^{c_{j}-2}+\cdots+1\right)} .
$$

The arguments of the zeros of $\frac{z^{\alpha}-1}{z-1}$ between 0 and $2 \pi$ are $2 k \pi / \alpha, 1 \leq k \leq \alpha-1$. So, by removing the constant $2 \pi$, the zeros of $\frac{z^{\alpha}-1}{z-1}$ can be identified with the ascending chain of rational numbers $1 / \alpha, 2 / \alpha, \ldots,(\alpha-1) / \alpha$. In this vein, for convenience, we denote

$$
[\alpha]=\left\{\frac{1}{\alpha}, \frac{2}{\alpha}, \ldots, \frac{\alpha-1}{\alpha}\right\} .
$$

When applying Fell's lemma, we will use an ascending chain of rational numbers as above instead of angle arguments. To connect this ascending chain with the zeros on $U$ in Fell's lemma, we need the definition below.

Definition 5. If $U$ is a finite multiset of complex numbers, write

$$
P_{U}(x)=\prod_{\alpha \in U}(x-\alpha) .
$$

If $U$ and $V$ are sets of real numbers, with no repeated elements, and moreover

$$
|U|=|V|=n, \quad U \cap V=\phi,
$$

we may write

$$
T:=U \cup V=\left\{t_{1}, t_{2}, \ldots, t_{2 n}\right\}
$$

with $t_{i}<t_{i+1}$ for all $i$. Define

$$
T_{1}=\left\{\left\{t_{1}, t_{2}\right\},\left\{t_{3}, t_{4}\right\}, \ldots,\left\{t_{2 n-1}, t_{2 n}\right\}\right\} .
$$

We say that a $U$-bad pair for $T$ or for $\left(P_{U}, P_{V}\right)$ is a pair of $T_{1}$ such that both elements belong to $U$; let $N_{U}(U, V)$ denote the number of $U$-bad pairs. The number of bad pairs is defined by

$$
N_{U}(U, V)+N_{V}(U, V) .
$$

Also a pair that is not bad is called a good pair.

How many zeros does $P_{r}(z)$ in (3) have on $U$ ? It is an easy consequence of Fell [1] that, if all elements of $\left[a_{1}\right] \cup\left[a_{2}\right] \cup\left[a_{3}\right]$ and $\left[b_{1}\right] \cup\left[b_{2}\right] \cup\left[b_{3}\right]$ with all commons deleted form good pairs, $P_{r}(z)$ has all its zeros on $U$. For example, we can prove

$$
F_{3,7,10} \hookrightarrow F_{5,6,9}
$$


We first observe that $c_{1}=5, c_{2}=3$ and $c_{3}=1$ from (2), which implies that $F_{3,7,10}$ and $F_{5,6,9}$ have common factor

$$
\left(z^{2}+z+1\right)\left(z^{4}+z^{3}+z^{2}+z+1\right) .
$$

So we now remove

$$
\frac{1}{3}, \frac{2}{3}, \frac{1}{5}, \frac{2}{5}, \frac{3}{5}, \frac{4}{5}
$$

once from both sets in ascending order

$$
[3] \cup[7] \cup[10]=\left\{\frac{1}{10}, \frac{1}{7}, \frac{2}{10}, \frac{2}{7}, \frac{3}{10}, \frac{1}{3}, \frac{4}{10}, \frac{3}{7}, \frac{5}{10}, \frac{4}{7}, \frac{6}{10}, \frac{2}{3}, \frac{7}{10}, \frac{5}{7}, \frac{8}{10}, \frac{6}{7}, \frac{9}{10}\right\}
$$

and

$$
[5] \cup[6] \cup[9]=\left\{\frac{1}{9}, \frac{1}{6}, \frac{1}{5}, \frac{2}{9}, \frac{3}{9}, \frac{2}{6}, \frac{2}{5}, \frac{4}{9}, \frac{3}{6}, \frac{5}{9}, \frac{3}{5}, \frac{6}{9}, \frac{4}{6}, \frac{7}{9}, \frac{4}{5}, \frac{5}{6}, \frac{8}{9}\right\},
$$

respectively, so that we have the remained elements

$$
\begin{aligned}
([3] \cup[7] \cup[10])^{\prime} & :=\left\{\frac{1}{10}, \frac{1}{7}, \frac{2}{7}, \frac{3}{10}, \frac{3}{7}, \frac{5}{10}, \frac{4}{7}, \frac{7}{10}, \frac{5}{7}, \frac{6}{7}, \frac{9}{10}\right\}, \\
([5] \cup[6] \cup[9])^{\prime}: & =\left\{\frac{1}{9}, \frac{1}{6}, \frac{2}{9}, \frac{2}{6}, \frac{4}{9}, \frac{3}{6}, \frac{5}{9}, \frac{4}{6}, \frac{7}{9}, \frac{5}{6}, \frac{8}{9}\right\} .
\end{aligned}
$$

These also have common element $1 / 2$ and there is a common factor $z+1$. After deleting $1 / 2$ from the both sets above, we form the pairs from one element of each set as following:

$$
\begin{aligned}
& \left(\frac{1}{10}, \frac{1}{9}\right),\left(\frac{1}{7}, \frac{1}{6}\right),\left(\frac{2}{9}, \frac{2}{7}\right),\left(\frac{3}{10}, \frac{2}{6}\right),\left(\frac{3}{7}, \frac{4}{9}\right), \\
& \left(\frac{5}{9}, \frac{4}{7}\right),\left(\frac{4}{6}, \frac{7}{10}\right),\left(\frac{5}{7}, \frac{7}{9}\right),\left(\frac{5}{6}, \frac{6}{7}\right),\left(\frac{8}{9}, \frac{9}{10}\right) .
\end{aligned}
$$

The pairs above are all good, and hence by Fell's lemma the polynomial

$$
\begin{aligned}
& (1-r) F_{3,7,10}+r F_{5,6,9} \\
= & (z+1)\left(z^{2}+z+1\right)\left(z^{4}+z^{3}+z^{2}+z+1\right) \\
& \left(z^{10}+z^{8}+r z^{7}+z^{6}+z^{5}+z^{4}+r z^{3}+z^{2}+1\right) .
\end{aligned}
$$

has all its zeros on $U$. As a by-product of this proof, the fact that the zeros of the polynomial

$$
z^{10}+z^{8}+r z^{7}+z^{6}+z^{5}+z^{4}+r z^{3}+z^{2}+1, \quad 0 \leq r \leq 1,
$$

lie on $U$ is obtained. 


\section{Remarks}

The relation $\sim$ is obviously reflexive and symmetry on the set $\mathcal{F}_{u, n}$. So it is natural to ask whether $\sim$ is transitive or not. If it is transitive, it is an equivalence relation and we may research further with this. Suppose $F_{a_{1}, a_{2}, a_{3}} \hookrightarrow F_{b_{1}, b_{2}, b_{3}}$ and $F_{b_{1}, b_{2}, b_{3}} \hookrightarrow F_{c_{1}, c_{2}, c_{3}}$ on $\mathcal{F}_{3, n}$. One might choose a polynomial $G_{r}(z)$ to hold $F_{a_{1}, a_{2}, a_{3}} \sim F_{c_{1}, c_{2}, c_{3}}$ as

$$
G_{r}(z)=(1-r)^{2} F_{a_{1}, a_{2}, a_{3}}+r(1-r) F_{b_{1}, b_{2}, b_{3}}+r F_{c_{1}, c_{2}, c_{3}}
$$

from the case $r=s$ of

$$
(1-s)\left[(1-r) F_{a_{1}, a_{2}, a_{3}}+r F_{b_{1}, b_{2}, b_{3}}\right]+s F_{c_{1}, c_{2}, c_{3}} .
$$

Many experimentations with computer algebra yield that $G_{r}(z)$ in (4) seems to have all its zeros on $U$ and so $F_{a_{1}, a_{2}, a_{3}} \sim F_{c_{1}, c_{2}, c_{3}}$. But the ideas of Fell allow us to make a counterexample. It follows from $F_{a_{1}, a_{2}, a_{3}} \hookrightarrow F_{b_{1}, b_{2}, b_{3}}$ and Fell's lemma that all zeros of the self-reciprocal polynomial

$$
A_{r}(z):=(1-r) F_{a_{1}, a_{2}, a_{3}}+r F_{b_{1}, b_{2}, b_{3}} \quad(0 \leq r \leq 1)
$$

are on $U$ and each zero is located on an open arc of $U$, where all such arcs are disjoint. We use notation [ ] as before, and assume that all sets [ ] below are in ascending order. Suppose that there are no elements of $\cup_{i=1}^{3}\left[c_{i}\right]$ between four consecutive elements of $\cup_{i=1}^{3}\left(\left[a_{i}\right] \cup\left[b_{i}\right]\right)$ that form two good pairs for $\left(\bigcup_{i=1}^{3}\left[a_{i}\right], \bigcup_{i=1}^{3}\left[b_{i}\right]\right)$, and two pairs for $\left(\bigcup_{i=1}^{3}\left(\left[a_{i}\right] \cup\left[b_{i}\right]\right), \bigcup_{i=1}^{3}\left[c_{i}\right]\right)$ just before and after such four consecutive elements of $\cup_{i=1}^{3}\left(\left[a_{i}\right] \cup\left[b_{i}\right]\right)$ are good. Then this yields at least one bad pair from the zeros of (4) and so not all zeros of (4) locate on $U$. For example, we let

$$
\left(a_{1}, a_{2}, a_{3}\right)=(5,19,21), \quad\left(b_{1}, b_{2}, b_{3}\right)=(8,17,20), \quad\left(c_{1}, c_{2}, c_{3}\right)=(11,16,18) .
$$

Then we have

$$
\bigcup_{i=1}^{3}\left(\left[a_{i}\right] \cup\left[b_{i}\right]\right)=\left\{\ldots, \frac{2}{5}, \frac{7}{17}, \frac{8}{19}, \frac{9}{21}, \ldots\right\}
$$

in ascending order on the set $(0,1)$, where the element $2 / 5$ actually occurs twice so that it is a zero of (5). Hence for our purpose we may convention that $(2 / 5,2 / 5)$ is a good pair for $\left(\bigcup_{i=1}^{3}\left[a_{i}\right], \bigcup_{i=1}^{3}\left[b_{i}\right]\right)$. We now can compute that

$$
\left(\frac{7}{17}, \frac{8}{19}\right)
$$

is a good pair, and all elements $<2 / 5$ and $\geq 9 / 21$ also form good pairs from the zeros of (5). Thus by Fell's lemma, each good pair above contains exactly one zero of (5). But

$$
\bigcup_{i=1}^{3}\left[c_{i}\right]=\left\{\ldots, \frac{7}{18}, \frac{7}{16}, \ldots\right\}
$$


in ascending order, and we see that

$$
\frac{7}{18}<\frac{2}{5}, \quad \frac{8}{19}<\frac{7}{16}
$$

This yields one bad pair from the zeros of (4). Thus

$$
F_{a_{1}, a_{2}, a_{3}} \nsim F_{c_{1}, c_{2}, c_{3}} .
$$

\section{References}

[1] H. J. Fell, On the zeros of convex combinations of polynomials, Pacific J. Math. 89 (1980), no. 1, 43-50.

[2] S. H. Kim, The zeros of certain family of self-reciprocal polynomials, Bull. Korean Math. Soc. 44 (2007), no. 3, 461-473.

[3] T. Sheil-Small, Complex Polynomials, Cambridge Studies in Advanced Mathematics 75, Cambridge University Press, Cambridge, 2002.

Department of Mathematics

SOOKMYUNG WOMEN'S UNIVERSITY

SEOul 140-742, KoreA

E-mail address: shkim17@sookmyung.ac.kr 